\title{
Development of a Genetic and Clinical Data-Based (GC) Risk Score for Predicting Survival of Hepatocellular Carcinoma Patients After Tumor Resection
}

\author{
Shengsen Chen Chao Wang An Cui Kangkang Yu Chong Huang \\ Mengqi Zhu Mingquan Chen
}

Department of Infectious Diseases and Hepatology, Huashan Hospital, Fudan University, Shanghai, China

\section{Key Words}

Cpt1a • PPARA $・$ Hepatocellular carcinoma $・$ Risk scoring system $・$ Prognosis

\begin{abstract}
Background/Aims: Carnitine palmitoyltransferase $1 \mathrm{~A}$ (CPT1A) is a rate-limiting enzyme in the transport of long-chain fatty acids for $\beta$-oxidation. Increasing evidence has indicated that CPT1A plays an important role in carcinogenesis. However, the expression and prognostic value of CPT1A in hepatocellular carcinoma (HCC) have not been extensively studied. Methods: Here, we collected 66 post-operative liver cancer tissue samples. Gene profile expression was tested by RT-PCR. Receiver operating characteristic (ROC) analysis was performed and multivariate analysis with Cox's Proportional Hazard Model was used for confirming the selected markers' predictive efficiency for HCC patients' survival. A simple risk scoring system was created based on Cox's regression modeling and bootstrap internal validation. Results: Cox multivariate regression analysis demonstrated that CPT1A, tumor size, intrahepatic metastasis, TNM stage and histological grade were independent risk factors for the prognosis of HCC patients after surgery. Our genetic and clinical data-based (GC) risk scoring system revealed that HCC patients whose total score $\geq 3$ are more likely to relapse and die than patients whose total score $<3$. Finally, the good discriminatory power of our risk scoring model was validated by bootstrap internal validation. Conclusions: The genetic and clinical data-based risk scoring model can be a promising predictive tool for liver cancer patients' prognosis after operation.
\end{abstract}




\section{Introduction}

Liver cancer constitutes a major health problem worldwide. Hepatocellular carcinoma (HCC) is the predominant form of primary liver cancer and is the third leading cause of tumor-related deaths globally $[1,2]$, accounting for over half a million deaths annually [3]. Therapeutic strategies such as surgical resection, chemotherapy and radiotherapy are available now, but the prognosis of patients with HCC still remains poor, mainly due to the absence of early symptoms and speedy tumor progression and invasion during the early stages [4-6]. Tumor occurrence, development and metastatic potential are frequently linked to the alteration of gene expression; therefore, it is imperative to identify the potential biological markers for early diagnosis [7], novel therapeutic strategies and prognosis prediction in patients with HCC $[8,9]$.

There is increasing evidence that altered cellular lipid metabolism is a hallmark of various cancers [10].. In contrast to normal cells using exogenous fatty acids, many cancer cells show high rates of de novo lipid synthesis [11]. Most current research has focused on lipogenesis, with the relevance of fatty acid oxidation (FAO) to cancer receiving less attention. Lately, the importance of FAO in cancer metabolism is being increasingly recognized. Since proliferating cancer cells require lipid for cellular membrane synthesis and other essential functions, reduced lipid catabolism in cancer cells can sustain rapid cell proliferation. Consistently, a previous study based on whole-genome microarray analysis has shown that genes involved in FAO are downregulated in HCC tissues [12]. Furthermore fatty acid incorporation into an oxidative pathway is reduced in five aggressive cancers compared to their non-aggressive counterparts [13].

It has been well documented that key enzymes involved in hepatic FAO and metabolism are influenced by carnitine palmitoyltransferase1 (CPT1) $[14,15]$. CPT1 is located in the outer mitochondrial membrane and facilitates the transport of long-chain fatty acids into the mitochondria for $\beta$-oxidation by converting them from acyl-CoA into acyl-carnitine [16]. In the liver, CPT1A is the primary isoform expressed while CPT1B and CPT1C mainly distribute into muscle, heart, and brain $[17,18]$. Previous studies have reported that CPT1A is downregulated in HCC tissues, due to downregulation of peroxisome proliferator-activated receptor $\alpha(\mathrm{PPAR} \alpha)[19]$. Meanwhile, Jibin Li et al. found that downregulation of CPT1A and PPAR $\alpha$ lead to the suppression of FAO, resulting in HCC growth and metastasis [19]. However, the specific association between prognosis of liver cancer patients and expression of CPT1A still remains unknown. Therefore, we hypothesized that CPT1A could be used as a pathological and prognostic biomarker for HCC patients, and conducted this study to elucidate the influence of CPT1A on clinical outcomes in human HCC.

\section{Materials and Methods}

\section{Patient cohorts}

Sixty-six patients (52 males, 14 females) from Huashan Hospital (Shanghai, China) were included in this study. Patient ages ranged from 15 to 85 years (mean \pm standard deviation [SD], 52.85 \pm 12.35 years). All the patients underwent radical hepatic resection for HCC, and the criteria for these resection procedures have been published [20]. None of the patients in this study received any preoperative chemotherapy or embolization therapy. Tumor tissue and adjacent non-tumor tissue were collected from the patients above and cryopreserved. The distance between adjacent non-tumor tissue and tumor tissue boundary was $2 \mathrm{~cm}$, beyond which was regarded as distant normal tissue. The selected tumor areas had more than $80 \%$ of tumor cells as being confirmed by histology examination. Tumor-node-metastasis (TNM) stage was classified according to the 7th edition of the AJCC (American Joint Committee on Cancer) cancer staging manual [21].

We have obtained the written informed consent from all patients. Experiments and procedures were in accordance with the Helsinki Declaration of 1975, and approved by the Human Ethics Committee of Shanghai Fudan University.

\section{KARGER}




\section{Cellular Physiology Cell Physiol Biochem 2018;48:491-502 and Biochemistry Published online: July 17, $2018 \quad \begin{aligned} & \text { DOI: 10.1159/000491779 } 2018 \text { The Author(s). Published by S. Karger AG, Basel } \\ & \text { www.karger.com/cpb }\end{aligned}$}

Chen et al.: Developing a GC Predicting Score for HCC Prognosis

Follow-up

All post-operative patients were subjected to regular surveillance as outpatients with standard protocol including 2-6 monthly computed tomography (CT) scan imaging of the liver. Any suspected intrahepatic recurrence was confirmed by hepatic angiography, post-lipiodol CT scan, and if necessary, percutaneous fine-needle aspiration cytology. According to the revised Response Evaluation Criteria in Solid Tumors (RECIST) guidelines (version 1.1)[22], the appearance of one or more new malignant lesions on multiphase CT scan or magnetic resonance (MR) imaging denotes disease progression.

Disease-free survival (DFS) was defined as the time period from the date of surgery operation to the first cancer recurrence (local or distant). Overall survival (OAS) was calculated from the date of cancer resection to death or the last contact. Follow-up ended at death or lost to follow-up or the fifth year, whichever came first.

\section{RNA extraction and RT-PCR assay}

Total RNA was extracted from human tissue samples with TRIzol Reagent (Invitrogen). The quality of RNA was examined by NanoDrop 1000 (Wilmington, DE., USA). Reverse transcription was performed using $1 \mu \mathrm{g}$ of isolated RNA with High Capacity cDNA Reverse Transcription kit (SYBR qPCR RT Mix, FSQ-101, TOYOBO). RT-PCR was performed in triplicate using SYBR Green Master Mix Kit and ABI 7500 Real-Time PCR System (Applied Biosystems, Foster City, CA, USA). Glyceraldehyde-3- phosphate dehydrogenase (GAPDH) was used as an internal control of RNA integrity. The $2^{-\Delta \Delta c t}$ method was used to analyze the relative changes in genes expression from real-time PCR experiments [23]. The primers for the genes were synthesized by Sangon Biotech Co., Ltd. (for all online suppl. material, see www.karger.com/doi/10.1159/000491779, primer sequences and annealing temperatures are shown in Table S1).

\section{Statistical analysis}

CPT1A or PPAR $\alpha$ expression differences between two groups were analyzed by Mann-Whitney U-test. Categorical variables were summed up as counts and compared by Fisher's exact test. We measured the area under the curve (AUC) of the receiver operating characteristic (ROC) curve for the two genes in order to calculate gene-expression cutoff value. Then the multivariate analysis with Cox's proportional hazard model was performed to further confirm the authenticity and validity of the three markers' predictive efficiency for HCC patients' prognosis. A simple risk score devised by using significant variables obtained from Cox's regression analysis with $\mathrm{P}<0.05$. The discrimination capability of the simple risk score was also presented by ROC curve. Finally, the Cox's Proportional Hazard Model in which risk score included was used for predicting the hazard trends of HCC recurrence and patients' death after surgery.

The discriminant analysis is a multivariable statistical method of classification, and the classification of a case is based on the combination of prior probabilities with discriminant functions. Therefore, the discriminant analysis was performed to further confirm the predictive power of the risk score system for predicting prognosis of HCC patients. The bootstrapping method (1000 simulations) was applied to evaluate the internal validation. All statistical tests were two-sided, and P values less than 0.05 were considered as statistically significant. The statistical analyses were performed by using SPSS version 21.0 and GraphPad Prism version 5.0.

\section{Results}

Correlation analysis between gene expression and clinical factors

A total of 66 patients were enrolled during the study period. We observed correlations between CPT1A expression and clinical markers, including tissue type (tumor tissue or nontumor tissue), tumor size, histological grade, intrahepatic metastasis, tumor stage, diseasefree survival time and overall survival time. However, the differences of patient's gender, age, hepatitis B surface antigen (HBsAg) expression, hepatitis B e antigen (HBeAg) expression, $\alpha$-fetoprotein (AFP) levels, lesion location and hepatic cirrhosis did not appear to have any correlation with CPT1A expression (see online suppl. material, Table S2). Then the HCC patients were grouped by tissue type (tumor tissue group vs non-tumor tissue group), tumor size ( $\geq 5 \mathrm{~cm}$ vs. $<5 \mathrm{~cm}, 5 \mathrm{~cm}$ was considered as cutoff value according to Hwang's study), $\mathrm{HBsAg}$ 
Fig. 1. Clinicopathologic features and expression of CPT1A. (A) The expression of CPT1A in HCC tissues and adjacent non-tumor tissues was determined by RT- PCR. (B) The expression of CPT1A mRNA in HBsAg negative and positive groups. (C) The expression of CPT1A mRNA in tumor size $\geq 5 \mathrm{~cm}$ and $<5 \mathrm{~cm}$ groups. (D) The expression of CPT1A mRNA in different histological grade (three-tier grading scheme) of primary HCC tissues. (E) The expression of CPT1A mRNA in intrahepatic metastasis and non intrahepatic metastasis groups. (F) The expression of CPT1A mRNA in different TNM stage of primary HCC tissues.

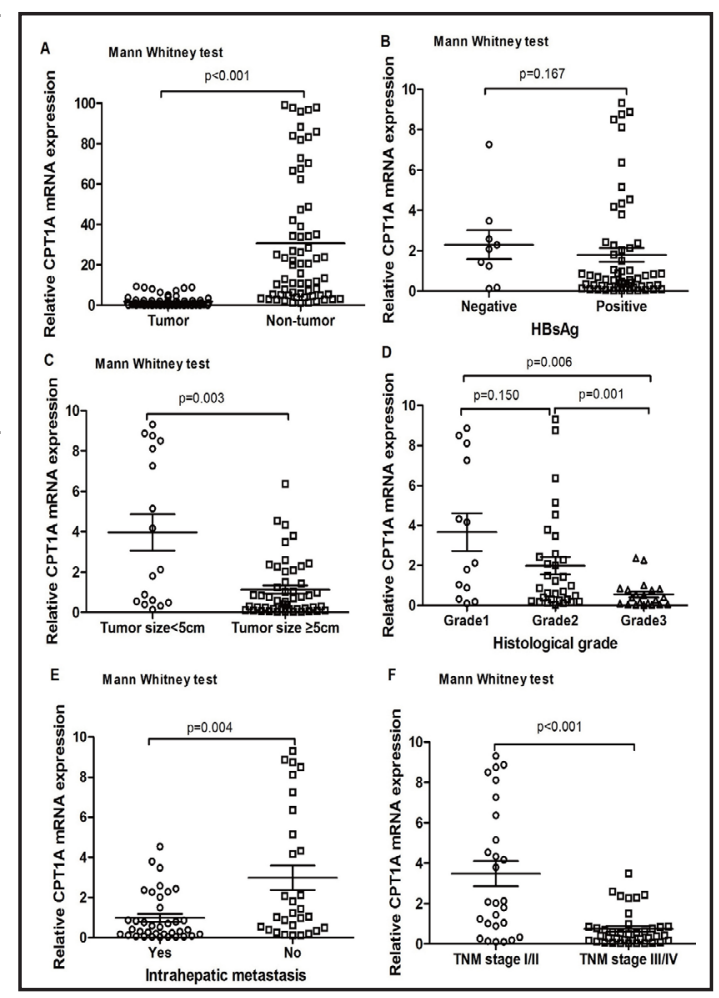

level(positive vs. negative), histological grade(divided into grade1, 2 and 3 groups) , tumor stage (TNM stage I/II vs. III/IV) and intrahepatic metastasis (yes vs. not) respectively. Thus we can further confirm the difference of CPT1A expression in these groups above (Fig. 1). CPT1A expression was significantly downregulated in human primary HCC tissues when compared with adjacent non-tumor tissues (Fig. 1A).

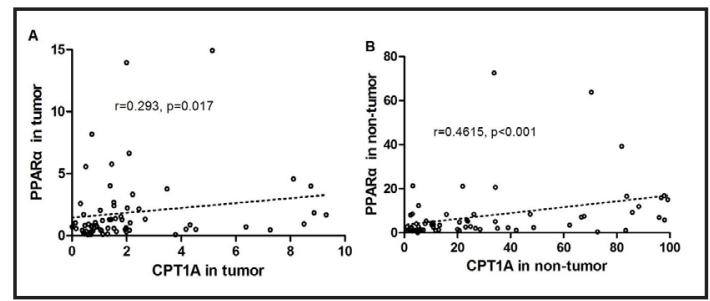

Fig. 2. The correlation between CPT1A levels and PPAR $\alpha$ levels in tumor (A) and non-tumor (B). The expression of CPT1A mRNA was also significantly related to tumor size ( $p=0.003$, Fig. $1 \mathrm{C}$ ), histological grade (grade1 vs. grade3, $\mathrm{p}=0.006$; grade 2 vs. grade $3, \mathrm{p}=0.001$; Fig. 1D), intrahepatic metastasis $(\mathrm{p}=0.004$, Fig. 1E) and TNM stage ( $p<0.001$, Fig. 1F); nevertheless, no significant differences in CPT1A levels were observed between HBsAg-positive and HBsAg-negative patients (Fig. 1B).

As a transcription factor, PPAR $\alpha$ plays a critical role in hepatic lipid oxidation mainly through regulating canonical target gene CPT1A [24, 25]. Spearman correlation analysis was performed between CPT1A and PPAR $\alpha$, and the result showed that a positive correlation existed between CPT1A and PPAR $\alpha$ both in tumor tissue(Fig. 2A) and non-tumor tissue(Fig. 2B). Similarly, when compared with adjacent non-tumor tissues, down-regulated expression of PPAR $\alpha$ also can be seen in tumor tissue (Fig. 3A). Decreasing PPAR $\alpha$ levels were also associated with higher TNM stages (Fig. 3F). In addition, the levels of PPAR $\alpha$ in tumor tissue varied between HBsAg-positive and HBsAg-negative patients (Fig. 3B), but showed no correlation with other parameters including tumor size (Fig. 3C), histological grade (Fig. 3D) and intrahepatic metastasis (Fig. 3E), which is a little different from the results for CPT1A. Also, gender, age, HBeAg, AFP, lesion location, and hepatic cirrhosis did not affect the PPAR $\alpha$ expression (see online suppl. material, Table S2). Furthermore, we also evaluated the relationship between tumor size and other factors in liver cancer patients, detailed results 
Fig. 3. Clinicopathologic features and expression of PPAR $\alpha$. (A) The expression of PPAR $\alpha$ in HCC tissues and adjacent non-tumor tissues was determined by RT- PCR. (B) The expression of PPAR $\alpha$ mRNA in HBsAg negative and positive groups. (C) The expression of PPAR $\alpha$ mRNA in tumor size $\geq 5 \mathrm{~cm}$ and $<5 \mathrm{~cm}$ groups. (D) The expression of PPAR $\alpha$ mRNA in different histological grade (three-tier grading scheme) of primary HCC tissues. (E) The expression of PPAR $\alpha$ mRNA in intrahepatic metastasis and non intrahepatic metastasis groups. (F) The expression of PPAR $\alpha$ mRNA in different TNM stage of primary HCC tissues.

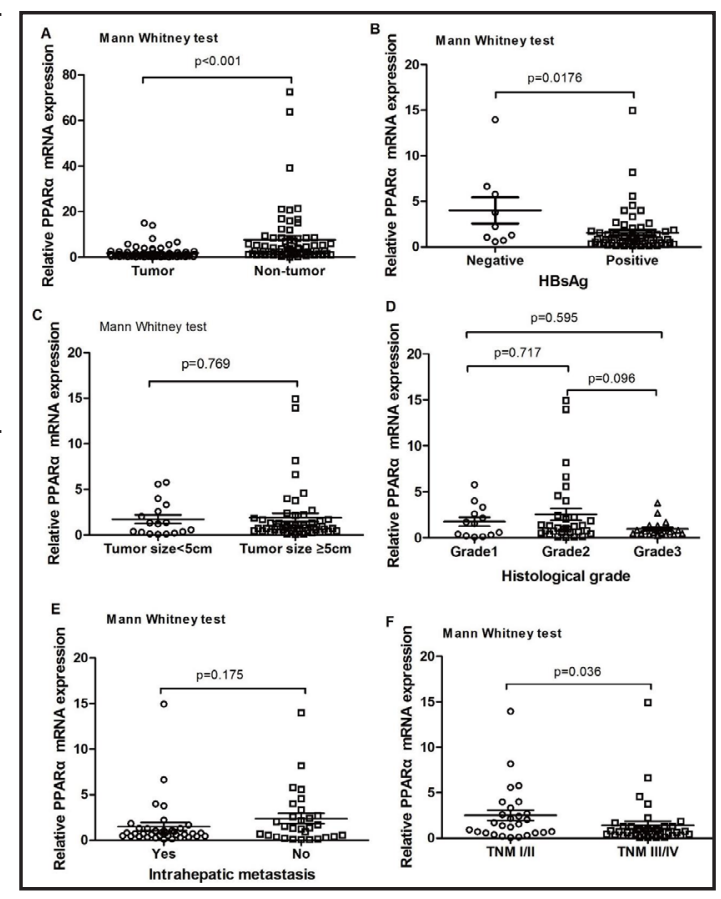

can be seen (see online suppl. material), in Table S3.

Clinical factors associated with prognosis of HCC patients

Clinical and demographic characteristics of the patients shown in Table 1 , reveal that recurrence and death all correlated with tumor size, histological grade, TNM stage and intrahepatic metastasis. Interestingly, HBsAg levels (positive vs. negative) influenced patients' death only and seemed to have not any relationship with HCC recurrence; however, the differences of patients' gender, age (60 years old was taken as cutoff value according to Gokcan's study [26]), HBeAg levels, AFP levels(100 ng/dl was identified as cutoff value based on the Brian's research [27]), hepatic cirrhosis, and tumor location did not appear to have any correlation with prognosis (recurrence and death). We then tested CPT1A and PPAR $\alpha$ expression in 66 samples to better understand the relationships between their expression
Table 1. Demographic and clinical characteristics of the subjects enrolled in the study (univariate analysis for HCC patients' prognosis). HBsAg, hepatitis B surface antigen; HBeAg, hepatitis B e antigen; AFP, alpha fetoprotein; $\mathrm{n}$, the sample number; Histological grade, according to the three-tier grading scheme; TNM stage, tumor-node-metastasis, according to the 7th edition of the AJCC (American Joint Committee on Cancer) cancer staging manual; $\mathrm{P}$ value according to the Fisher exact test

\begin{tabular}{|c|c|c|c|c|c|c|}
\hline Variable & Non Recurrence & Recurrence & $\mathrm{P}$ & Non death & Death & $\mathrm{P}$ \\
\hline Gender (male), n(\%) & $26(76.5)$ & $26(81.3)$ & 0.766 & $21(80.8)$ & $31(77.5)$ & 0.771 \\
\hline Age ( $\geq 60$ years), n(\%) & $10(29.4)$ & $8(25.0)$ & 0.785 & $8(30.8)$ & $10(25.0)$ & 0.778 \\
\hline Tumor size $(\geq 5 \mathrm{~cm}), \mathrm{n}(\%)$ & $20(58.8)$ & $29(90.6)$ & 0.004 & $14(53.8)$ & $35(87.5)$ & 0.002 \\
\hline Histological grade, $\mathrm{n}(\%)$ & & & $<0.001$ & & & $<0.001$ \\
\hline Grade 1 & $12(35.3)$ & $1(3.1)$ & & $12(46.2)$ & $1(2.5)$ & \\
\hline Grade 2 & $21(61.8)$ & $11(34.4)$ & & $14(53.8)$ & $18(45.0)$ & \\
\hline Grade 3 & $1(2.9)$ & $20(62.5)$ & & $0(0)$ & $21(52.5)$ & \\
\hline TNM stage, $n(\%)$ & & & $<0.001$ & & & $<0.001$ \\
\hline Stage I & $10(29.4)$ & $0(0)$ & & $10(38.5)$ & $0(0)$ & \\
\hline Stage II & $12(35.3)$ & $5(15.6)$ & & $12(46.2)$ & $5(12.5)$ & \\
\hline Stage III & $9(26.5)$ & $15(46.9)$ & & $3(11.5)$ & $21(52.5)$ & \\
\hline Stage IV & $3(8.8)$ & $12(37.5)$ & & $1(3.8)$ & $14(35.0)$ & \\
\hline HBsAg(positive), n(\%) & $26(76.5)$ & $31(96.9)$ & 0.028 & $20(76.9)$ & $37(92.5)$ & 0.138 \\
\hline HBeAg(positive), n(\%) & $13(38.2)$ & $8(25.0)$ & 0.297 & $10(38.5)$ & $11(27.5)$ & 0.422 \\
\hline $\operatorname{AFP}(\geq 100$ ng/dl), n(\%) & $17(50.0)$ & $18(56.3)$ & 0.631 & $14(53.8)$ & $21(52.5)$ & 0.915 \\
\hline Intrahepatic metastasis(yes),n(\%) & $11(32.4)$ & $26(81.3)$ & $<0.001$ & $5(19.2)$ & $32(80.0)$ & $<0.001$ \\
\hline Hepatic cirrhosis(yes), n(\%) & $14(41.2)$ & $12(37.5)$ & 0.805 & $9(34.6)$ & $17(42.5)$ & 0.610 \\
\hline Tumor location, n(\%) & & & 0.078 & & & 0.681 \\
\hline Left lobe of liver & $13(38.2)$ & $5(15.6)$ & & $7(26.9)$ & $11(27.5)$ & \\
\hline Right lobe of liver & $15(44.1)$ & $16(50.0)$ & & $13(50.0)$ & $18(45.0)$ & \\
\hline Both lobe of liver & $4(11.8)$ & $10(31.3)$ & & $4(15.4)$ & $10(25.0)$ & \\
\hline Both lobe at the junction & $2(5.9)$ & $1(3.1)$ & & $2(7.7)$ & $1(2.5)$ & \\
\hline
\end{tabular}


and prognosis status. The two markers were significantly overexpressed in non-recurrence group (PPAR $\alpha, p=0.010$; CPT1A, $p<0.001$ ) (Fig. 4A) and non-death group (PPAR $\alpha, p=0.031$; CPT1A, p<0.001) (Fig. 4B) compared with their counterpart groups.

\section{Differentiating power of CPT1A and PPAR $\alpha$ for the prognosis of liver cancer}

In order to determine the differentiating power of CPT1A and PPAR $\alpha$ for the prognosis of HCC patients after tumor resection, we finally resorted to ROC curve analyses by individually using the expression of each marker (CPT1A and PPAR $\alpha$ ). It can be seen from the ROC curves that CPT1A showed higher AUC than PPAR $\alpha$ for HCC recurrence (CPT1A, AUC=0.788; PPAR $\alpha$, AUC=0.685; Fig. 4C) and patients' death (CPT1A, AUC=0.881; PPAR $\alpha, A U C=0.658$; Fig. 4D). The optimal cutoff values of the two genes expression levels were determined to maximize the sum of sensitivity and specificity, the detailed can be seen in Fig. 4C and 4D. Therefore, patients were further categorized into two groups (low expression vs. high expression) based on the cutoff values of the two genes expression levels (see online suppl. material, Table S4).

\section{Multivariable analysis with Cox proportional hazards model}

After that, the multivariate Cox's proportional hazard model, in which the factors such as CPT1A, PPAR $\alpha$, tumor size, intrahepatic metastasis, tumor stage, histological grade and HBsAg were respectively included, was performed to deeply investigate the independent prognostic factors for patients' survival. The results of the multivariable analysis showed that low expression of CPT1A was significantly correlated with poor DFS (HR=5.192, p=0.001) and OAS (HR=5.818, $\mathrm{p}<0.001)$. Additionally, tumor size, intrahepatic metastasis, TNM stage and histological grade were also independent risk factors for the prognosis of HCC patients (Table 2). It's interesting that PPAR $\alpha$ (DFS, $\mathrm{p}=0.087$; OAS, $\mathrm{p}=0.449$ ) and HBsAg (DFS, $\mathrm{p}=0.292 ;$ OAS, $\mathrm{p}=0.866$ ) levels did not appear to have any associations with patients' survival.

\begin{tabular}{|c|c|c|c|c|c|c|}
\hline \multirow[b]{2}{*}{ Parameters } & \multicolumn{3}{|c|}{ Disease-free survival } & \multicolumn{3}{|c|}{ Overall survival } \\
\hline & $\beta$ & HR(95\%Cl) & $P$ & $\beta$ & $\mathrm{HR}(95 \% \mathrm{Cl})$ & $\mathrm{P}$ \\
\hline \multicolumn{7}{|l|}{ Genes level in liver tumor tissue } \\
\hline CPT1A expression(low vs. high) & 1.647 & $5.192(1.902-14.174)$ & 0.001 & 1.761 & $5.818(2.189-15.462)$ & $<0.001$ \\
\hline PPAR $\alpha$ expression(low vs. high) & 1.017 & $2.765(0.863-8.857)$ & 0.087 & 0.321 & $1.378(0.601-3.159)$ & 0.449 \\
\hline Tumor size ( $\geq 5 \mathrm{~cm}$ vs. $<5 \mathrm{~cm}$ ) & 1.588 & $4.892(1.456-16.434)$ & 0.010 & 1.261 & $3.529(1.355-9.183)$ & 0.011 \\
\hline Intrahepatic metastasis(yes vs. no) & 1.422 & $4.147(1.513-11.370)$ & 0.006 & 1.315 & $3.727(1.534-9.052)$ & 0.004 \\
\hline TNM stage(III / IV vs. I / II) & 1.814 & $6.137(1.868-20.165)$ & 0.003 & 2.267 & $9.651(3.459-26.930)$ & $<0.001$ \\
\hline Histological grade( 3 vs. 1 and 2 ) & 1.707 & $5.510(2.337-12.989)$ & $<0.001$ & 1.089 & $2.971(1.467-6.018)$ & 0.002 \\
\hline HBsAg(positive vs. negative) & 1.129 & $3.094(0.379-25.250)$ & 0.292 & 0.119 & $1.127(0.282-4.504)$ & 0.866 \\
\hline
\end{tabular}

Fig. 4. Expression levels of CPT1A and PPAR $\alpha$ in the groups with different prognoses and ROC analyses of the two genes for predicting the prognosis of HCC patients. (A) Expression levels of CPT1A and PPAR $\alpha$ in the groups with recurrence vs. non recurrence. (B) Expression levels of CPT1A and PPAR $\alpha$ in death and non death groups. (C) and (D) The ROC curves of CPT1A and PPAR $\alpha$ for differentiating the HCC recurrence and death.

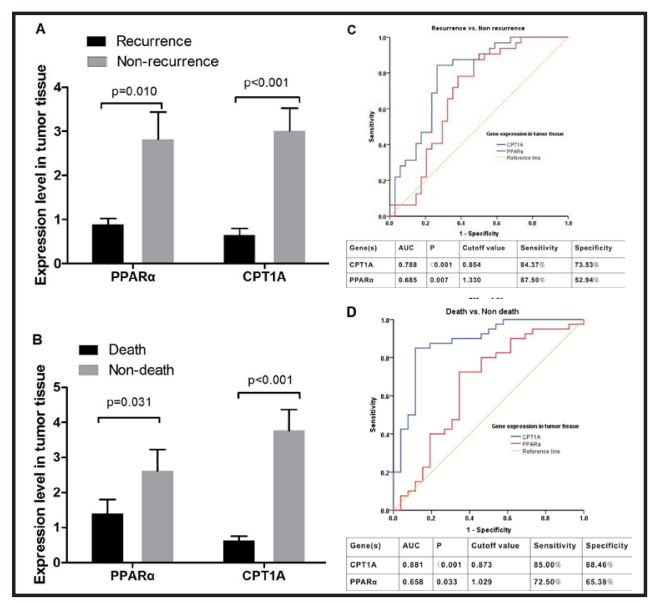


Development of a new biomarkerbased risk score for predicting the HCC patients' prognosis

The development of the new risk score and selection of the prognostically most important clinical variables and biomarkers was based on the results of multivariate analysis of Cox's proportional hazard model. The most important predictors of HCC patients' survival were the significant variables in the Cox model with $\mathrm{P}<0.05$ (CPT1A, tumor size, intrahepatic metastasis, TNM stage, histological grade). The score of these variables were defined as the quotient (rounded to nearest integer) of corresponding estimated beta coefficients from a Cox's regression analysis divided by the smallest absolute value of regression coefficient in the same Cox model (see online suppl. material, Table S5 and Table S6, the section of algorithm for score system (see online suppl. material), in supplementary material provide the detailed procedures of risk score development). Total score was the sum of each variable score and DFS was evaluated by the total score ranged from 0 to 5 (see online suppl. material, Table S7). Similarly, OAS was predicted by the total score ranged from 0 to 7 (see online suppl. material, Table S8). The final score was named GC risk score based on inclusion of gene (CPT1A) and clinical data (tumor size, intrahepatic metastasis, TNM stage and histological grade).

The prognosis statuses of HCC patients were taken as final diagnosis, and the total score was considered as diagnostic index. Then two ROC curves were plotted to assess the efficiency of the scoring system for predicting patients' prognosis. Area under the curve for evaluating DFS was 0.886 (Fig. $5 \mathrm{~A}$ ) and for assessing OAS was 0.962 (Fig. 5B).The optimal cutoff points of the two ROC curves both were score 3 for HCC recurrence and death. For clinical and informative application, patients were further categorized into two risk groups (total score, $<3$ vs. $\geq 3$ ) to evaluate DFS and OAS. Furthermore, as shown from Fig. 6, HCC patients whose total score more than 3 were more likely to relapse and die than patients whose score less than 3.

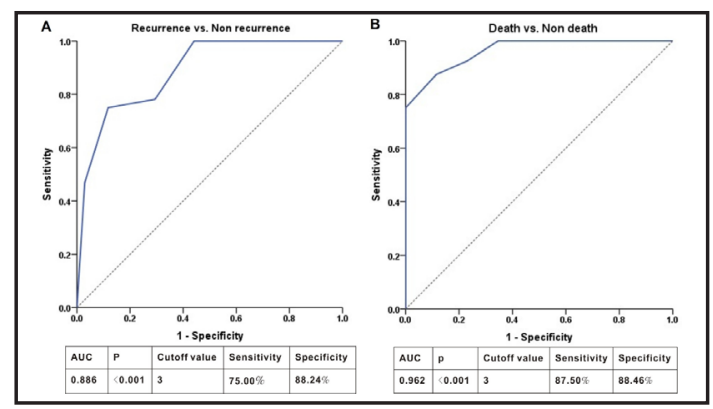

Fig. 5. ROC curves with simplified risk score to distinguish HCCs' recurrence (A) and death (B).

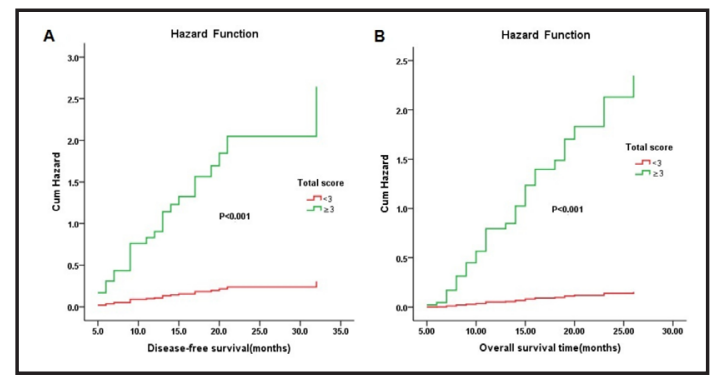

Fig. 6. The impact of total scoring system on diseasefree survival (A) and overall survival (B) with Cox's regression analysis; $p$ values were confirmed with Cox proportional hazards model.

Table 3. Classification table of discriminant analysis. Predictive power of CPT1A, tumor size, intrahepatic metastasis, TNM stage and histological grade for predicting the prognosis of HCC patients. This procedure is designed to develop a set of discriminating functions which can help predict death vs. non death and recurrence vs. non recurrence based on these independent risk factors; 66 cases were used to develop a model to discriminate among the death vs. non death and recurrence vs. non recurrence; five predictive variables were entered. The 66 observations were used to fit the model, $89.4 \%$ and $84.8 \%$ were correctly classified

\begin{tabular}{lllll}
\hline Actual prognosis & Group size(n) & Predicted prognosis \\
\hline \multirow{5}{*}{ Non death } & 26 & Non death(n) & Death(n) & Correct percentage \\
Death & 46 & 7 & 0 & $100.0 \%$ \\
Overall percentage & & & 33 & $82.5 \%$ \\
& & Recurrence(n) & Non recurrence(n) & $89.4 \%$ \\
Recurrence & 32 & 26 & 6 & $81.3 \%$ \\
Non recurrence & 34 & 4 & 30 & $88.2 \%$ \\
Overall percentage & & & & $84.8 \%$ \\
\hline
\end{tabular}




\section{Cellular Physiology Cell Physiol Biochem 2018;48:491-502 \begin{tabular}{l|l} 
DOI: 10.1159/000491779 & O 2018 The Author(s). Published by S. Karger AG, Basel \\
www.karger.com/cpb
\end{tabular}}

GC risk scoring model confirmation and validation

Subsequently, the predictive power of GC prognosis risk score included CPT1A, tumor size, intrahepatic metastasis, TNM stage and histological grade for discerning recurrence and death was also confirmed by discriminant analysis. It can be clearly seen from Table 3 that the overall predictive percentage of $89.4 \%$ and $84.8 \%$ respectively for OAS and DFS. Also, more importantly, it correctly classified $82.5 \%$ of the samples in the death group and $81.3 \%$ of the samples in the recurrence group. The discriminant analysis was made for each patient and the detailed information was shown(see online suppl. material) in Table S9 and Table S10. Finally, the discriminative ability of risk scoring model was validated by using the biascorrected AUC with 1000-sample bootstrapping. This prediction scoring model showed good discriminatory performance among the 1000 bootstrap internal samples (Fig. 7).

\section{Discussion}

Cancer cells take advantage of altered lipid metabolism for cell membrane biogenesis, growth and survival $[28,29]$. Though the pro-oncogenic role of lipogenesis is well defined, the biological relevance of lipid catabolism via FAO to cancer has not been well characterized. Although various metabolomic and pharmacological studies have suggested involvement of FAO in cancer cells [30-33], there is no direct evidence or molecular mechanism supporting the concept that cancer cells indeed rely on FAO for maintaining malignancy. As a ratelimiting enzyme in the $\beta$-oxidation of long-chain fatty acids, the expression levels of CPT1A impacts FAO, leading to the alteration in cell lipid metabolism [34]. Huanjie Shao et al. have reported that $\mathrm{CPT} 1 \mathrm{~A}$ is abundantly expressed in ovarian cancer, which contributes to poor prognosis of the disease [35]. Additionally, increased levels of CPT1A has been associated with high grade glioblastoma [36]. Paradoxically, CPT1A has been shown to be downregulated in the cytosolic compartment of human colorectal and breast cancer tissues and in several neoplastic cell lines [37]. There is evidence that FAO is suppressed in hepatocellular carcinomas via downregulating CPT1A expression [19], however the utility of changes in CPT1A levels and prognosis has yet to be investigated thoroughly.

In our study, we found that the CPT1A mRNA expression was significantly decreased in majority of primary HCCs that we examined compared with non-tumor liver tissues in the same patients (Fig. 1A). We further investigated the correlations between CPT1A expression and clinicopathologic features of liver cancer and observed CPT1A expression was significantly correlated with tumor size and intrahepatic metastasis (Fig. 1C and 1E). Compared with late tumor stage and poor histologic grade HCC patients, we found that early TNM stage and benign differentiation in histology seemed to be associated with high expression of CPT1A (Fig. 1D and 1F). PPAR $\alpha$ is highly expressed in tissues displaying a high metabolic rate of fatty acids (such as the liver and skeletal muscle) and modulates intracellular lipid metabolism by transcriptional regulation of CPT1A involved in mitochondrial fatty acid oxidation [38, 39]. We observed that in tumor and non-tumor groups an increase in PPAR $\alpha$ expression correlates with upregulation of CPT1A (Fig. 2). Similarly, our results suggested a significantly lower level of PPAR $\alpha$ expression in HCC tissue samples than in noncancerous tissue samples (Fig. 3A), and PPAR $\alpha$ expression was significantly correlated with TNM stage (Fig. 3F). However, unlike CPT1A, no significant associations were found between PPAR $\alpha$ expression 
in cancerous tissues and primary clinicopathological parameters of HCC, including tumor size (Fig. 3C), histological grade (Fig. 3D) and intrahepatic metastasis(Fig. 3E). In addition, there was also a fact that PPAR $\alpha$ expression was significantly different between positive and negative HBsAg groups (Fig. 3B). These data above suggests that the expression of CPT1A might be regulated by other factors, other than PPAR $\alpha$.

Subsequently, we further investigated the impact of PPAR $\alpha$ and CPT1A expression on liver patients' prognosis after tumor resection. As shown from Fig. 4A and 4B, HCC patients with lower levels of PPAR $\alpha$ and CPT1A would be apt to relapse and die than those with higher levels of the two genes. To determine the predictive power of PPAR $\alpha$ and CPT1A for predicting HCC patients's prognosis, ROC curves were generated and the results identified CPT1A, but not PPAR $\alpha$, as useful predictive biomarker. The AUCs of PPAR $\alpha$ and CPT1A to distinguish between recurrence and non-recurrence were 0.685 and 0.788 (Fig. 4C), and to differentiate between death and non- death were 0.658 and 0.881(Fig. 4D). Therefore, they alone or in combination can be used to evaluate survival of HCC patients. Apart from the influence of PPAR $\alpha$ and CPT1A on prognosis of HCC patients, relationships between other clinical factors and prognosis was fuzzily confirmed by Fisher's exact test, the results was summarized in Table 1, which showed that tumor size, histological grade, TNM stage and intrahepatic metastasis were significantly correlated with recurrence and death. Interestingly, HBsAg was merely relevant with HCC recurrence and showed no relationship with patients' death. Additionally, gender, age, HBeAg levels, AFP levels, hepatic cirrhosis, and tumor location were roughly identified as irrelevant factors for the prognosis of HCC patients after operation.

Since Fisher's exact test could hardly manage the interference existed among these variables above, a multivariate analysis must be performed to identify the authenticity and validity of the prognostic factors detected from the Table 1. According to the results of Fisher's exact test and ROC analysis, ultimately, Cox multivariate regression model was performed included the factors such as PPAR $\alpha$, CPT1A, tumor size, histological grade, TNM stage, intrahepatic metastasis and HBsAg. The data demonstrated that CPT1A, tumor size, histological grade, TNM stage and intrahepatic metastasis were independent risk factors for the prognosis of HCC patients after operation. As shown in Table 2, the group with CPT1A low expression may have 5.192 times risk of liver cancer recurrence and 5.818 times risk of patients' death compared with the gene high expression group. We also could observe from Table 2 that patients with tumor size $\geq 5 \mathrm{~cm}$ and intrahepatic metastasis were more likely to relapse and die, the hazard ratios of recurrence were 4.892 and 4.147 , and the hazard ratios of death were 3.529 and 3.727 respectively. Besides, patients with TNM stage III/IV have 6.137 times risk of tumor recurrence and 9.651 times risk of death compared to their counterparts with TNM stage I/II. Furthermore, we found that the group with histological grade 3 appeared to have about 5.510 times risk of liver cancer recurrence and 2.971 times risk of death compared with group of histological grade 1 and 2. However, PPAR $\alpha$ and HBsAg seemed uncorrelated with DFS and OAS in the Cox regression analysis. In our present study, only 66 HCC patients were included, which could hide the statistical significance of some variables in the Cox regression analysis due to the small sample size. Therefore, a large sample is needed in the future research to ensure the authenticity and stability of the results.

In order to deeply investigate the impact of CPT1A, tumor size, histological grade, TNM stage and intrahepatic metastasis on DFS and OAS, we developed a simple score (named GC score-gene and clinical data) composed of these five variables to predict HCC prognosis after tumor resection. The strong predictive power of scoring system to evaluate prognosis can be described by the ROC curves (recurrence, AUC=0.886, Fig. 5A; death, AUC=0.962, Fig. 5B). Also, the cutoff values of scores were determined by ROC curves. Patients with prediction score of $<3$ vs. $\geq 3$ had distinctly different risk of HCC recurrence and patients' death (Fig. 6). Identification of patients' risk for their prognosis could initiate an individualized surveillance program for HCC patients after tumor resection. Ultimately, for the purpose of further confirming the predictive efficiency of the prediction score including CPT1A, tumor size, histological grade, TNM stage and intrahepatic metastasis, a discriminant analysis was

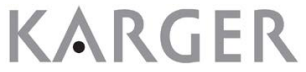


made for each patient enrolled in this study. The discriminant analysis made up of CPT1A, tumor size, histological grade, TNM stage and intrahepatic metastasis demonstrated a very strong overall predictive value for evaluating HCC recurrence (overall percentage: $84.8 \%$ ) and patients' death (overall percentage: $89.4 \%$ ). It is noteworthy that discriminant analysis showed a correct classification of $82.5 \%$ samples in the death group and $81.3 \%$ samples in the recurrence group (Table 3). Based on the discriminant analysis, the posterior probability of prognosis resulted to range between $50 \%$ and $100 \%$ for all cases, few classification errors occurred when the posterior probability was higher than $80 \%$ (see online suppl. material, Table S9 and Table S10). Finally, the prediction model was validated internally by bootstrap resampling (Fig. 7). Hence, applying the gene-based risk score as a case classifier strengthens its importance as post-operative predictor for the prognosis of HCC patients.

In conclusion, our study has provided evidence that CPT1A could serve as a useful, novel biomarker for prognosis in liver cancer. We have observed decreased expression of CPT1A in liver tumor tissue from patients with resected HCCs, and that these changes in expression appear to correlate with tumor size, histological grade, intrahepatic metastasis and TNM stage. Multivariable analysis with Cox's proportional hazard model identified that CPT1A, tumor size, histological grade, TNM stage and intrahepatic metastasis were independent risk factors for the prognosis of HCC patients after tumor resection. Additionally, a novel risk score for predicting HCC patients' survival using one biomarker (CPT1A) and information on tumor size, histological grade, TNM stage and intrahepatic metastasis, named GC-risk score (gene, clinical data), was developed in this study and finally validated internally by bootstrap resampling. The genetic and clinical data-based risk score acted as predictive model firstly used in our study and may be a promising predictive tool for liver cancer patients' prognosis after operation.

\section{Acknowledgements}

This work was supported by grants from the National Natural Science Foundation of China (No. 81773088). We also thank Luis Cocka (postdoctoral fellow in University of Pennsylvania) help us to proofread and modify language errors in this manuscript.

\section{Disclosure Statement}

The authors declare to have no competing financial interests.

\section{References}

$\rightarrow 1$ Luo HL, Chen J, Luo T, Wu FX, Liu JJ, Wang HF, Chen M, Li LQ, Li H: Downregulation of MacrophageDerived T-UCR uc.306 Associates with Poor Prognosis in Hepatocellular Carcinoma. Cell Physiol Biochem 2017;42:1526-1539.

-2 Huang R, Wang X, Zhang W, Zhangyuan G, Jin K, Yu W, Xie Y, Xu X, Wang H, Sun B: Down-Regulation of LncRNA DGCR5 Correlates with Poor Prognosis in Hepatocellular Carcinoma. Cell Physiol Biochem 2016;40:707-715.

-3 Siegel R, Naishadham D, Jemal A: Cancer statistics, 2013. CA Cancer J Clin 2013;63:11-30.

4 Cervello M, McCubrey JA, Cusimano A, Lampiasi N, Azzolina A, Montalto G: Targeted therapy for hepatocellular carcinoma: novel agents on the horizon. Oncotarget 2012;3:236-260.

5 Maluccio M, Covey A: Recent progress in understanding, diagnosing, and treating hepatocellular carcinoma. CA Cancer J Clin 2012;62:394-399.

6 Bruix J, Gores GJ, Mazzaferro V: Hepatocellular carcinoma: clinical frontiers and perspectives. Gut 2014;63:844-855. 


\section{Cellular Physiology Cell Physiol Biochem 2018;48:491-502 \begin{tabular}{l|l} 
DOI: 10.1159/000491779 & Ond Biochemistry \\
Published online: July 17, 2018 & $\begin{array}{l}\text { 2018 The Author(s). Published by S. Karger AG, Basel } \\
\text { www.karger.com/cpb }\end{array}$
\end{tabular}}

Chen et al.: Developing a GC Predicting Score for HCC Prognosis

7 Wang Y, Liu T, Tang W, Deng B, Chen Y, Zhu J, Shen X: Hepatocellular Carcinoma Cells Induce Regulatory T Cells and Lead to Poor Prognosis via Production of Transforming Growth Factor-beta1. Cell Physiol Biochem 2016;38:306-318.

8 Zhu AX, Duda DG, Sahani DV, Jain RK: HCC and angiogenesis: possible targets and future directions. Nat Rev Clin Oncol 2011;8:292-301.

-9 Yang F, Lv L, Zhang K, Cai Q Liu J, Jiang Y: Elevated FOXC2 Expression Promotes Invasion of HCC Cell Lines and is Associated with Poor Prognosis in Hepatocellular Carcinoma. Cell Physiol Biochem 2017;44:99-109.

10 Glaysher J: Lipid metabolism and cancer. Curr Opin Lipidol2013;24:530-531.

-11 Currie E, Schulze A, Zechner R, Walther TC, Farese RJ: Cellular fatty acid metabolism and cancer. Cell Metab 2013;18:153-161.

12 Wu JM, Skill NJ, Maluccio MA: Evidence of aberrant lipid metabolism in hepatitis C and hepatocellular carcinoma. HPB (Oxford) 2010;12:625-636.

13 Louie SM, Roberts LS, Mulvihill MM, Luo K, Nomura DK: Cancer cells incorporate and remodel exogenous palmitate into structural and oncogenic signaling lipids. Biochim Biophys Acta 2013;1831:1566-1572.

14 Keung W, Ussher JR, Jaswal JS, Raubenheimer M, Lam VH, Wagg CS, Lopaschuk GD: Inhibition of carnitine palmitoyltransferase-1 activity alleviates insulin resistance in diet-induced obese mice. Diabetes 2013;62:711-720.

15 Setoyama D, Fujimura Y, Miura D: Metabolomics reveals that carnitine palmitoyltransferase-1 is a novel target for oxidative inactivation in human cells. Genes Cells 2013;18:1107-1119.

-16 Schreurs M, Kuipers F, van der Leij FR: Regulatory enzymes of mitochondrial beta-oxidation as targets for treatment of the metabolic syndrome. Obes Rev 2010;11:380-388.

17 McGarry JD, Brown NF: The mitochondrial carnitine palmitoyltransferase system. From concept to molecular analysis. Eur J Biochem 1997;244:1-14.

18 Price N, van der Leij F, Jackson V, Corstorphine C, Thomson R, Sorensen A, Zammit V: A novel brainexpressed protein related to carnitine palmitoyltransferase I. Genomics 2002;80:433-442.

19 Li J, Huang Q Long X, Zhang J, Huang X, Aa J, Yang H, Chen Z, Xing J: CD147 reprograms fatty acid metabolism in hepatocellular carcinoma cells through Akt/mTOR/SREBP1c and P38/PPARalpha pathways. J Hepatol 2015;63:1378-1389.

20 Nagasue N, Kohno H, Chang YC, Taniura H, Yamanoi A, Uchida M, Kimoto T, Takemoto Y, Nakamura T, Yukaya H: Liver resection for hepatocellular carcinoma. Results of 229 consecutive patients during 11 years. Ann Surg 1993;217:375-384.

-21 Edge SB, Compton CC: The American Joint Committee on Cancer: the 7th Edition of the AJCC Cancer Staging Manual and the Future of TNM. Ann Surg Oncol 2010;17:1471-1474.

22 Eisenhauer EA, Therasse P, Bogaerts J, Schwartz LH, Sargent D, Ford R, Dancey J, Arbuck S, Gwyther S, Mooney M, Rubinstein L, Shankar L, Dodd L, Kaplan R, Lacombe D, Verweij J: New response evaluation criteria in solid tumours: revised RECIST guideline (version 1.1). Eur J Cancer 2009;45:228-247.

-23 Livak KJ, Schmittgen TD: Analysis of relative gene expression data using real-time quantitative PCR and the 2(-Delta Delta C(T)) Method. Methods 2001;25:402-408.

24 Bocher V, Pineda-Torra I, Fruchart JC, Staels B: PPARs: transcription factors controlling lipid and lipoprotein metabolism. Ann N Y Acad Sci 2002; 967:7-18.

25 Latruffe N, Vamecq J: Peroxisome proliferators and peroxisome proliferator activated receptors (PPARs) as regulators of lipid metabolism. Biochimie 1997;79:81-94.

26 Gokcan H, Savas N, Oztuna D, Moray G, Boyvat F, Haberal M: Predictors of Survival in Hepatocellular Carcinoma Patients. Ann Transplant 2015;20:596-603.

27 Carr BI, Guerra V: Low Alpha-Fetoprotein Levels Are Associated with Improved Survival in Hepatocellular Carcinoma Patients with Portal Vein Thrombosis. Digest Dis Sci 2016;61:937-947.

28 Menendez JA, Lupu R: Fatty acid synthase and the lipogenic phenotype in cancer pathogenesis. Nat Rev Cancer 2007;7:763-777.

29 Kuhajda FP: Fatty acid synthase and cancer: new application of an old pathway. Cancer Res 2006;66:59775980.

-30 Nieman KM, Kenny HA, Penicka CV, Ladanyi A, Buell-Gutbrod R, Zillhardt MR, Romero IL, Carey MS, Mills GB, Hotamisligil GS, Yamada SD, Peter ME, Gwin K, Lengyel E: Adipocytes promote ovarian cancer metastasis and provide energy for rapid tumor growth. Nat Med 2011;17:1498-1503. 


\section{Cellular Physiology Cell Physiol Biochem 2018;48:491-502

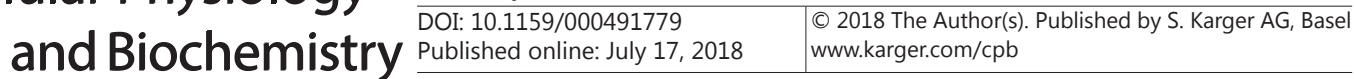 \\ Chen et al.: Developing a GC Predicting Score for HCC Prognosis}

31 Wu X, Daniels G, Lee P, Monaco ME: Lipid metabolism in prostate cancer. Am J Clin Exp Urol 2014;2:111120.

-32 Carracedo A, Cantley LC, Pandolfi PP: Cancer metabolism: fatty acid oxidation in the limelight. Nat Rev Cancer 2013;13:227-232.

-33 Linher-Melville K, Zantinge S, Sanli T, Gerstein H, Tsakiridis T, Singh G: Establishing a relationship between prolactin and altered fatty acid beta-oxidation via carnitine palmitoyl transferase 1 in breast cancer cells. Bmc Cancer 2011;11:56.

-34 Akkaoui M, Cohen I, Esnous C, Lenoir V, Sournac M, Girard J, Prip-Buus C: Modulation of the hepatic malonyl-CoA-carnitine palmitoyltransferase 1A partnership creates a metabolic switch allowing oxidation ofde novo fatty acids1 Biochem J 2009;420:429-438.

-35 Shao H, Mohamed EM, Xu GG, Waters M, Jing K, Ma Y, Zhang Y, Spiegel S, Idowu MO, Fang X: Carnitine palmitoyltransferase $1 \mathrm{~A}$ functions to repress FoxO transcription factors to allow cell cycle progression in ovarian cancer. Oncotarget 2016;7:3832.

36 Cirillo A, Di Salle A, Petillo O, Melone MA, Grimaldi G, Bellotti A, Torelli G, De’ SM, Cantatore G, Marinelli A, Galderisi U, Peluso G: High grade glioblastoma is associated with aberrant expression of ZFP57, a protein involved in gene imprinting, and of CPT1A and CPT1C that regulate fatty acid metabolism. Cancer Biol Ther 2014;15:735-741.

37 Mazzarelli P, Pucci S, Bonanno E, Sesti F, Calvani M, Spagnoli LG: Carnitine palmitoyltransferase I in human carcinomas: a novel role in histone deacetylation? Cancer Biol Ther 2007;6:1606-1613.

-38 Staels B, Fruchart JC: Therapeutic roles of peroxisome proliferator-activated receptor agonists. Diabetes 2005;54:2460-2470.

-39 Staels B, Dallongeville J, Auwerx J, Schoonjans K, Leitersdorf E, Fruchart JC: Mechanism of action of fibrates on lipid and lipoprotein metabolism. Circulation 1998;98:2088-2093. 